(Aus dem II. anatomischen Institute zu Berlin.)

\title{
Ein Beitrag zur Kenntniss des menschlichen Primordialcraniums ${ }^{1}$ ).
}

Von

\section{Martin Jacoby.}

Hierzu Tafel $V$.

Die Morphologie des Kopfes, zu der die Morphologie des Schädels sich im Laufe der Ietzten Jahrzehnte entwickelt hat, nimmt eine bevoryugte Stellung in der Forschung ein. Indem ich es unterlasse, die grosse Literatur zu besprechen, die sich mit diesem wichtigen und so interessanten Thema beschäftigt, will ich nur auf die wesentlichsten und die neueren Arbeiten über das Primordialcranium der Sänger und insbesondere des Menschen eingehen, da meine eigenen Untersuchungen nur das Primordialcranium des Menschen behandeln.

Nachdem bereits $R$ athke und $R$ eichert auf viele wichtige Punkte hingewiesen hatten, erfuhr die Entwicklungsgeschichte des Säugerschädels und speciell auch des mensehlichen Craniums wesentliche Bereicherung durch die Untersuchungen $\mathrm{K} \ddot{0} 1 \mathrm{lik \text {er's }}$ und seiner Schüler.

Köllike r betonte die ausserordentliche Reduction des menschlichen Primordialcraniums gegenüber dem der Säuger. Er constatirte, dass eine primordiale Decke überhaupt nicht zur Ausbildung gelangt und dass Seitenwände nur theilweise angelegt werden.

1) Die Veranlassung zur Bearbeitung des vorliegenden Themas gab eine, von der Berliner medicinischen Fakultät ausgeschriebene Preisaufgabe: „Die erste Anlage des Primordialcraniums menschlicher Embryonen ist $\%$ untersuchen und die Form desselben durch Reconstruction genau festzustellen." Der von mir eingereichten Abhandlung, welcher ein durch Reconstruction hergestelltes Modell des Primordialcraniums beigefügt war, wurde der ausgeschriebene Preis zuerkannt. Das Modell wird in nächster Zeit von Herrn Z ie gler in Freiburg in Wachs ausgeführt und vervielfältigt werden. 
Unter Kölliker's Leitung beschrieb Spöndli die Primordialcrania mehrerer Säugethiere und des Menschen. Seine Untersuchungen machten es bereits möglich, sich eine im allgemeinen richtige Vorstellnng von der Gestalt des Primordialcraniums zu bilden. Den Schwerpunkt legte er in seinen Ausfïhrungen auf die Bekämpfung der a l t e n Wirbeltheorie des Schädels, die damals erst zu wanken begann und beschäftigte er sich des weiteren mit der Verknöcherung des Primordialeraniums.

Geraume Zeit später setzte Decker Spöndli's Untersuchungen fort. Er verbreitete sich über eine ganze Anzahl Säugethiere, untersuchte aber nicht das menschliche Cranium. Mit Hülfe der von ihm fast aussehliesslich angewandten makroskopischen Untersuchungsmethoden stellt er einige Unterschiede zwischen den verschiedenen Species fest und betonte besonders, dass das knorpelige Primordialcranium zu einer Zeit, in der noch keine primordialen Knochenkerne aufgetreten sind, durchaus noch keine Abgrenzung einzelner Theile unter sich erkennen lässt, sondern dass vielmehr die aneinanderstossenden lartieen unmittelbar ineinander übergehen.

Endlich kommen für das Primordialcranium speciell des Menschen die Arbeiten von $\mathrm{Dursy}, \mathrm{Hannover}$ und v. N o orden in Betracht.

D u rsy hat neben vielem Anderen das eigenthümliche Verhalten der Chorda in der Schädelanlage untersucht und die Unterschiede, die in dieser Beziehung zwischen den Säugethieren und dem Menschen bestehen, hervorgehoben. Ausserdem verweilt er ausfiilrlich bei der Entwicklung des Nasenskelets und legt mit Recht auf die Thatsache Nachdruck, dass im Primordialcranimm ein continuirlicher Uebergang von dem Prinordialknorpel des Keilbeins bis zum Nasenknorpel besteht.

Hannover hat eine grosse Anzahl menschlicher Embryonen untersucht, ohne jedoch in Bezug auf die genaue Gestalt und die Ausbildnng des Craniums wesentlich neues beizubringen. Er hat zur Erkenntniss der Formen sich hauptsächlich der makroskopischen Präparationsmethoden bedient und das Mikroskop und die Schnittmethoden nur für histologische Fragen benutzt. Eingehender hat er die älteren Stadien untersucht und die Verknöcherung des Schädels und die daran sich anknüpfenden Theorien einer genaueren Erörterung unterzogen. 
v. Noorden ist bisher der Einzige, der das Primordialcranium des Menschen mit den neuesten Methoden untersucht hat. Er hat 3 Embryonen an Serienschnitten studirt und Reconstructionen angefertigt. Für das Studium des Primordialcraniums des Menschen in der Zeit da es am Besten ausgebildet ist, waren seine Embryonen aber ohne Zweifel viel zu wenig entwickelt.

Wie ich glaube, ist hier sicher noch eine Lïcke in unserer Kenutniss des Primordialeraniums auszufüllen und ich folgte daher gern einer Anregung des Herrn Professor Hertwig, mich an die Bearbeitung der von der Fakultät gestellten Aufgabe zu machen.

Für die gütige Anregung, die freundliche Ueberlassung des Materiales und der Hilfsmittel seines Institutes sowie für die jeder Zeit bereitwilligst ertheilten werthvollen Rathschläge fühle ich mich ihm zu grösstem Danke verpflichtet.

Zunächst soll nun einiges ïber das Material und die ausgeübten Metboden hier seinen Platz finden, dann werde ich die genaue Gestalt des Primordialcraniums beschreiben, wie sie sich nach meinen Befunden darstellt und zum Schluss werde ich die Ergebnisse der Autoren discutiren und die meinigen kurz zusammenfassen.

\section{Material und Methode.}

Mein Material bestand aus einem menschlichen Embryo von $30 \mathrm{~mm}$ Scheitelsteisslänge. Genaues luber die Methode seiner Behandlung findet sich in einer Arbeit von B a umgarten, der an ihm die Entwicklung der Gehörknöchelchen studirt hat. Hier will ich nur das wichtigste anführen. Der gut conservirte Embryo war in Boraxkarmin durchgefärbt und in eine Serie von $0,02 \mathrm{~mm}$ dicken Schnitten zerlegt worden, die vom Scheitel bis zur Herzgegend geführt wurden, die Schnitte wurden mit Bleu de Lyon nachgefärbt.

Die Methode ermöglicht, wie $\mathrm{B}$ a u $\mathrm{mg}$ a r t e n angiebt, nicht nur eine ausgezeichnet deutliche Färbung und Differenzirung des Knorpelgewebes, sondern giebt auch klare Bilder des Faserverlaufes im Centralnervensystem, die Ganglienzellenkerne erscheinen dunkelroth, die Nervenfasern hellblan. Ich will noch weiterhin hervorheben, dass auch die eben ange- 
legten Deckknochen durch eine andere Nuance des blauen Tones sich deutlich differenziren und dass die nicht unwichtigen Zellenanhäufungen im Bindegewebe durch intensivere Färbung hervortreten.

Der Embryo stellt ein günstiges Objekt zur Feststellung der genauen Gestalt des Primordialcraniums dar, weil das Letztere bereits hinreichend entwickelt ist und man da, wo die Entwicklung noch nicht abgeschlossen ist, doch riemlich deutlich den Weg erkennen kann, den die Entwicklung nebmen wird. Der Embryo ist alter and weiter entwickelt als die von v. Noorden untersuchten Embryonen.

Zum Studium der Gestalt des Craniums bediente ich mich nun neben der Schnittbetrachtung einer Reconstructionsmethode, die in der Hauptsache der B o r'n'schen Plattenmethode entspricht.

Die Schnitte wurden mittels eines Projectionsapparates unter Anwendung von electrischem Bogenlicht auf eine vertikal gerichtete Zeichenfläche projicirt und bei 25 facher Vergrösserung gezeichnet. Der Apparat vereinigt eine gute Lichtquelle mit grossem Gesichtsfeld bei beliebiger Vergrösserung. Mit jeder Zeichnung wurden zwei sich winklig schneidende Definirlinien mitge»eichnet; die Vergrösserung wurde nach jeder Unterbrechung der Arbeit genau controlirt.

Gezeichnet wurden die knorpligen Organe von dem distalen Ende der Meckel'schen Knorpel an bis dahin, wo scheitelwärts der Knorpel überhaupt aufhört. Auch die Deckknochen wurden berïcksichtigt, andere Organe nur zur Orientirung mit in die Zeichmung aufgenommen. Von den $0,02 \mathrm{~mm}$ dicken Schnitten wurde immer der zweite gezeichnet, im ganzen 168 Schnitte. Ich zeichnete auf Cartonpappe von $1 \mathrm{~mm}$ Dicke, so dass also die Vergrösserung in der Höhe der Flächenvergrösserung entsprach. Die Zeichnungen wurden dann unter dem Mikroskop noch einmal genau auf ihre Richtigkeit geprifft und schliesslich mit einer feinen Laubsäge ausgeschnitten.

\section{Gestalt des Primordialcraniums im allgemeinen.}

Bei der Betrachtung des Modells füllt zunächst die r a dimentäre Ausbildung des Primordialcraniums auf. Eine Decke ist überhaupt nicht vorhanden, Seitenwände sind nur im hinteren 
Theile ausgebildet. Nach hinten zu sehen wir in der Gegend der späteren Squama occipitis eine weite Oeffnung. Hier besteht der dorsale Verschluss noch nicht aus Knorpel, sondern aus der nicht in das Modell aufgenommenen „Membrana spinoso-occipitalis“. $\mathrm{Nur}$ in der Gegend, in der später sich die Grenze zwischen Parietale und Occipitale befindet, erkennen wir eine knorplige dorsale Wandung von geringer Höhe. Alle Knorpelanlagen, die bereits ausgebildet sind, stehen miteinander in Zusammenhang; wir können keine L ü $\mathrm{ck}$ en zwischen den einzelnen Anlagen des Craniums, wohl aber bei den meisten mit mehr oder weniger Schwierigkeiten Grenzen innerhalb des Knorpels erkennen.

\section{Nomenclatur.}

Wir werden nun bei der Beschreibung des Craniums im folgenden keine rationelle Nomenclatur anwenden können, weil das so lange eine Unmöglichkeit ist, als wir noch nicht uber die erste Anlage des menschlichen Primordialcraniums unterrichtet sind. Hat ja doch G a up erst neuerdings darauf hingewiesen, dass eine rationelle Nomenclatur, die 0 . Hertwig bereits in seiner Batrachierarbeit als erstrebenswerthes Ziel hingestellt hatte, sich zur Zeit noch nicht einmal für die so gut gekannten Amphibien durchführen lässt. Ich werde also, wie es hisher üblich war, die Namen von den späteren knöchernen Gebilden nehmen, jedoch mit der Kölliker'schen Schule von Regi onen spreehen, um erkennen zu lassen, dass wir nur Theile eines einheitlichen Ganzen vor uns haben.

\section{Topographischer Ueberblick.}

(Fig. 1 und 2.)

Als Kern des Craniums erkennen wir in der Mitte der Basis die Regio sphenoidalis mit den Alae temporales (A.t.) und orbitales (A.o.). Nach vorne geht die Regio sphenoidalis in die $R$ egi o e th moidalis über und diese wieder in das Septum narium, welches mit den seitlichen Nasenknorpeln (Fig. 2, Rn.) in Verbindung steht. Nach hinten setzt sich die Regio sphenoidalis mit schwer feststellbarer Grenze in die Regio occipitalis fort. Zu beiden Seiten des Hinterhaupts und des Keilbeinknorpels erblickt man die knorpligen Gelörkapseln (Fig. 1, Cchl.), die mit beiden auch 
in knorpliger Verbindung stehen. Nach oben gehen die Gehörkapseln in vertikal gestell te, gew ölbte Platten über, die, wie schon früher angedeutet, in ihren oberen Theilen in der Medianebene dorsalwärts zusammentreffen. Das Hinterhauptsbein geht ohne eigentliche Grenze in den obersten $\mathrm{Halsw}$ irbel uber; eine Abgrenzung lässt sich höchstens durch das Verhalten der Chorda vornehmen.

Vom Visceralskelet und von den Deckknochen, soweit sie schon vorhanden sind, wird einiges der Vollständigkeit halber angefugt werden.

\section{Regio sphonoidalls.}

Die Regio sphenoidalis steht mit den Gehörkapseln, dem Occipitalknorpel und der Regio ethmoidalis in knorpliger Verbindung. Wir unterseheiden an ihr einen $\mathrm{K} o \mathrm{rper}$ und $\mathrm{zwei}$ Fl i g e lpa a re.

$\mathrm{Körper.} \mathrm{Der} \mathrm{Körper} \mathrm{zeigt} \mathrm{in} \mathrm{der} \mathrm{Mitte} \mathrm{eine} \mathrm{Grube} \mathrm{mit}$ einem gegenüber dem umgebenden Knorpel sehr dünnen Boden. In dieser Grube, wohl ohne Zweifel der S ell a tu r ci ca (Fig. 1, St.), lagert ein Organ, das mit dem Zwisehenhirn in Verbindung steht, die Hy pophyse. Der düne Knorpelboden dentet wohl noch auf ein früher vorhandenes Hypophysenloch hin.

Die Hinterwand der Grube ist hoch und wird nach der freien oberen Kante hin allmählich dünner. Es handelt sich um das Dorsum ephippii (D.e.), an dem wir noch nichts vom Processus clinoidei postici bemerken. Auf dem Medianschnitte des Modells sehen wir etwa $12 \mathrm{~mm}$ unterhalb des freien Randes des Dorsum ephippii innerhalb des Knorpels die $\mathrm{Ch}$ orda dorsalis enden.

Nach hinten geht der Körper des Keilbeinknorpels als Clivus Blumenbachii (Cl. B.) ziemlich steil in die Occipitalregion uber. Vor der Sella tureica erhebt sich das Tuberculum ephippii (T.e.), vor dem das Chiasma nervorum opticorum (Cch.n.opt.) ruht. Processus clinoidei medii sind nicht vorbanden. Am hinteren Winkel des Körpers fällt der Can al is caroticus ( $I$ u. $I I)$ auf.

Wie zu erwarten, bildet der Keilbeinknorpel eine solide Masse; die Höhlen des erwachsenen Knochens beruhen ja erst auf später auftretenden Resorptionsprocessen. 
Alae sphenoidales. Im Gegensaty zu den definitiven knöchernen Verhältnissen sind die Alae orbitales (Fig. 1 und 2,A.o.) des Primordialcraniums bedeutend grösser als die Alae temporales $(A . t$.).

Die Ala temporalis ist an einer Knorpelbrücke befestigt, welche Keilbein mit Felsenbeinknorpel im Primordialcranium verbindet; der Schläfenflügel ragt frei in das umgebende Bindegewebe hinein, ohne jede sonstige knorplige Verbindung, er hat ungefahr die Gestalt einer sehr spitzen Pyramide, deren Basis nach oben gerichtet ist. Hier oben findet sich eine ziemlich tiefe Rinne, welche durch den zweiten Ast des Trigeminus ausgefüllt ist, wir haben wohl vermuthlich das noch nicht geschlossene Foramen rotundum vor uns. Ein Foramen ovale und spinosum ist nicht zu konstatiren, auch nicht zu erwarten, da wir den 3. Ast des Trigeminus ausserhalb des Knorpels seinen Weg einschlagen sehen, während wir eine Arteria meningea media überhaupt nicht sicher feststellen konnten.

A lae orbitales. Die Alae orbitales entspringen von dem vorderen Theile des Sphenoidalknorpels mit zwei Wurzeln, durch die ein Loch $(I I I)$ für den Durchtritt des Sehnerven gebildet wird. Die Flügel sind nach vorn und lateral gerichtet. Die Gestalt ist etwa sichelförmig, wenn man von den zwei Fortsützen, dic jeder Fliigel trägt, absicht.

Der eine Fortsat\% ist nach hinten gerichtet und endet als freier Vorsprung über dem vorderen Theile der Sella turcica. Wir erkennen die Processus clinoidei anteriores (IV).

Die anderen Fortsätze sind nach oben gerichtet und finden sich an der Stelle, an welcher die Sichel ilıre stärkste Krümmung zeigt. Für sie können wir ontogenetisch keine Erklärung finden, später werden wir sehen, dass die Phylogenie das Räthsel zu lösen fähig ist.

Vorn und unten finden wir eine Knorpelbrïcke zwischen der Ala orbitalis (Fig. 1 und 2, A.o.) und der Nasoethmoidalregion. Schon Dursy hat eine solche beobachtet und sie Orbitalplatte $(O p$.$) benannt.$

An die Flügel schliessen sich nach vorne hin an 2 flache Sehalen, deren Concavitait medianwärts gerichtet ist und die aus zarten Deckknochen bestehen: Die Frontalia (Fr.). Selbstverständlich haben sie iluren Platz in dem Bindegewebe, in dem 
sie sich entwickelt haben und sind dem Knorpel nur benachbart, nicht mit ihm in Verbindung.

\section{Regio ethmoidalis et nasalis (Rn.).}

Der Sphenoidalknorpel geht obne Grenze allmählich in den Ethmoidalknorpel über. Das Niveau der Ethmoidalregion erreicht nicht das der Ala orbitalis; sie setzt sich an etwas tiefer gelegene Schichten des vorderen Keilbeinendes an, wendet sich dann in leichtem, nach oben offenem Bogen nach vorne, um an dem Vorderende des Craniums eine wulstförmige Erhebung yu zeigen. Dieses ganze Gebilde ist als schmaler Kamm nur auf die Medianebene beschränkt. Wohl olme Zweifel haben wir in ihm die Crista galli (Cr.g.) zu suchen; knorplige Siebplatten fehlen noch gänzlich.

Wie das Modell zeigt, setzt sich die Ethmoidalregion nach unten in vertikaler Richtung in die Nasenscheidewand fort. Betrachten wir das Cranium von vorne, so sehen wir auf dem Vorderende der Nasenscheidewand eine ziemlich tiefe Furche, welche dadurch entsteht, dass hier die Seitentheile des Nasenknorpels sich mit vorspringendem Bogen an die Scheidewand ansetzen.

Die knorplige Nasenscheidewand zeigt ebenso wie das sie umgebende Bindegewebe eine dem frühen embryonalen Stadium entsprechende erhebliche Breite; an ihrem hinteren, freien Ende ist sie kolbig verdickt.

Von knorpligen $\mathrm{N}$ a se $\mathrm{nmuscheln}$ ist noch nicht viel zu berichten. Sie sind alle bereits angelegt, aber zeigen ausser der oberen erst eine sehr geringe Entwicklung. Die Stellen der seitlichen Nasenknorpelwände, an denen wir später die Muscheln finden werden, sehen wir verbreitert und etwas nach innen gebogen.

\section{Regio occipitalis.}

Wir sind jetzt genöthigt, den Blick über das Cranium wandern zu lassen und uns der hinten gelegenen Regio oceipitalis zuzuwenden. Die Occipitalregion steht mit der Regio sphenoidalis, R. petrosa und mit dem Halswirbelknorpel in Verbindung. Gegen die Sphenoidalregion hin lässt sich überhaupt keine Grenze konstruiren, gegen die Halswirbelsäule durch das Verhalten der Chorda dorsalis, gegen die Regio petrosa nur unter Würdigung 
der verschiedensten Verhältnisse. Auf diese Punkte kommen wir später zurück.

An der Regio occipitalis unterscheiden wir eine Pars basilaris und zwei Partes laterales, die sich alle um ein Foramen magnum gruppiren. Die Trennung der einzelnen Theile ist weniger durch scharfe Scheidung der Formen als durch histologische Ver'schiedenbeit bedingt.

\section{Gestalt der Occipitalregion.}

Auch hier werden wir am sehnellsten durch das Studium des Modells Klarheit gewinnen. Die Pars basilaris der Occipitalregion bildet bereits mit dem anstossenden Theil der Sphenoidalregion einen Cliv us, welcher nicht die gleiche Art der Steigung wie der knöcherne Clivus zeigt. In den unteren Partieen, welche dem Foramen magnum nahe. liegen, finden wir nämlich eine sehr s a n f te Steigung, während dann plötzlich etwa in der Mitte des Verlaufes eine sehr starke, fast senkrechte Steigung ihren Anfang nimmt.

Die Pars basilaris entspricht nach ihrer Lage und Gestalt ziemlich den definitiven Verhältnissen; die Partes lateralis, die nach v. Noorden's passendem Vergleich den Hälften einer Klappmuschel ähnlich sind, liefern die Partes condyloideae. Die Seitentheile werden in dorso-ventraler Richtung vom Canalis pro nervo hypoglosso (Fig. 1, IX) durchsetzt. Von ihnen aus entwickeln sich die primordialen Schuppenanlagen, die noch sehr unbedeutend sind, nach oben von den später zu besprechenden "Parietalplatten" überlagert sind und hinten sich nicht zum Ringe schliessen, sondern durch straffe Bindegewebszage verbunden erscheinen. Wir haben die Kölliker'sche ${ }_{n} M$ e mbran a spinosooc eipitalis" vor uns. Nach vorne und lateralwärts entsendet schliesslich noch jede Pars lateralis einen deutlichen „Processus jugularis".

Einiges über den histologischen Bau der Regio occipitalis.

Es wird jetzt nicht ohne Interesse sein, im Anschluss an die Beschreibung der Gestalt der Regio occipitalis auch einen Blick auf ihre histologischen Verhältnisse zu werfen, da nämlich die Scheidung zwischen Pars basilaris und Partes laterales im wesentlichen durch die Struktur bedingt ist. 
Schon bei schwacher Vergrösserung, ja schon wenn man die Schnitte gegen das Licht hält, fällt auf, dass das Lichtbrechungsvermögen der Partes laterales ein viel grösseres ist als das der Pars basilaris. Bei genauerer Beobachtung erkennt man dann, dass die Partes laterales aus reiferem Knorpel bestehen und dass die optischen Erscheinungen auf der starken Ausbildung der Grundsubstanz beruhen. Der Knorpel der Pars basilaris ist viel jünger und die Grundsubstanz ist hier weniger entwickelt. Am jüngsten ist der Knorpel der Processus jugulares. Bemerkenswerth ist, dass eine scharfe Trennung zwischen der Pars basilaris mit ihrem jüngeren Knorpel und der Partes laterales mit dem reiferen Knorpel durch diese histologischen Verhältnisse besteht. Dieser Umstand dürfte vielleicht für die Erkenntniss der ersten Anlage des Craniums zu verwerthen sein.

\section{Anlage der Halswirbelsăule.}

Wenn wir jetzt einiges über die Anlage der Halswirbelsäule anfügen, so geschieht das nur insoweit, als es zur Abgrenzung des Primordialcraniums gegen die Wirbelsäule nöthig ist.

Schon in der primordialen Halswirbelsäule nehmen Atlas und Epistropheus eine gesonderte Stellung ein, welche durch den Zahnfortsatz des Epistropheus bedingt ist.

An den unteren Wirbeln erkennen wir auf das Deutlichste das regelmässige Abwechseln von Wirbeln und Zwischenwirbelscheiben. An den Wirbeln können wir einen Körper, gut ausgebildete aber nicht dorsal geschlossene Seitentheile und schliesslich Verbindungsbrücken zwischen Körper und Seitentheilen unterscheiden. In der Nähe ihres lateralen Randes werden diese Brücken durch die Foramina transversaria unterbrochen.

Die Zwischenwirbelscheiben bestehen nur aus einer kreisförmigen Knorpelanlage, welche sich rings um die Chorda entwickelt hat. An ihren beiden Seiten verlassen die Nerven die Wirbelanlage.

Beim Atlas und Epistropheus finden wir nun die bekannten, eigenthümlichen Verhältnisse; aus dem Körper des Atlas ist bereits der Zahn des Epistropheus geworden. Ventral vom Zahnfortsatze sehen wir eine schmale Knorpelspange, welche die Seitentheile des Atlas verbindet. Es ist die von Froriep beim Menschen gesehene, aber erst bei Rindsembryonen genau erkannte 
hypochordale Knorpelspange, welche, wie er gezeigt hat, sich bei den Vögeln an allen Wirbeln findet.

\section{Chorda dorsalis.}

An die Betrachtung der Regio occipitalis und der Halswirbelsäule schliesst sich mit Nothwendigkeit das Studium der Chorda dorsalis. Es treten einem hier zweierlei Arten von Schwierigkeiten entgegen. Einmal sind die Verhältnisse am Kopfende der Chorda sehr verwickelte, andrerseits aber finden wir die Chorda in einem für die Untersuchung nicht gunstigen $\mathrm{Zu}$ stande. Auf vielen Schnitten erscheint die Chorda geschrumpft; sie füllt das Lumen, das der Knorpel frei lässt, nicht aus. Die Scheide ist oft zerrissen und hängt in Fetzen um die Chorda. Auf manchen Schnitten ist nur die Lücke im Knorpel erhalten, während die Chorda selbst tiberhaupt nicht sichtbar ist. Man könnte versucht sein, dieses Verhalten mit Dursy als ein durch die Entwicklung bedingtes anzusehen, wenn nicht zweierlei uns eines besseren belehren würde.

Zunächst finden wir auf einigen Schnitten die Chorda in gutem Zustande, schön umgeben von der Scheide, die Knorpellïcke gänzlich ausfüllend. Dieser Grund ist allein allerdings noch nicht stichhaltig; ja Froriep hat daraus sogar gerade den Schluss gezogen, dass es sich um keine postmortalen, durch die Methode der Bearbeitung verursachten Erscheinungen handeln kann. Es zeigt jedoch weiterhin eine einfache Ueberlegung und lehrt die Erfahrung, dass ein so zartes Gewebe wie die Chorda bei der Härtung stärker schrumpfen muss als der umgebende Knorpel und dass so das Entstehen von künstlichen Lücken und veränderten Bildern ein erklärlicher Vorgang ist. So ist denn auch die Chorda da wo wir sie im retropharyngealen Raum oder im Ligamentum suspensorium dentis nur von Bindegewebe umgeben antreffen, wohl erhalten.

Ich bin allerdings der Ansicht, dass die Bedingungen der Zerrung und sonstige ungtunstige Einflisse auf die in Knorpel eingeschlossene Chorda auch physiologisch bereits einwirken können und dass es sich in solchen Stadien nicht nur um postmortale Schrumpfung zu handeln braucht, welche $\mathrm{H}$ is mit vollem Recht bei sehr jugendlichen Embryonen immer für die Veränderungen verantwortlich macht. Die Ansicht von $H$ is ist 
mir um so einleuchtender, als ich bei einer Reihe von jugendlichen Süugerembryonen, die mit rasch wirkenden Flüssigkeiten fixirt und vortrefflich conservirt waren, die Chorda ohne jede Schrumpfung gut erhalten sah.

\section{Einiges über die Histologie der Chorda.}

Die Chorda stellt ein zellenreiches Organ dar; wir erkennen Zellgrenzen und intensiv gefärbte Zellkerne. Umgeben ist dieser Zellstrang von einer glashellen, strukturlosen Membran, der „inneren Chordascheide".

Um diese wiederum legt sich der Knorpel, der die skeletogene Chordascheide vorstellt und zwar finden wir centralwärts, also der Chorda zugewandt, reichliche Knorpelgrundsubstanz, um die sich dann concentrisch ein Zellenring herumlegt.

Froriep hat zwischen dem Knorpel und der inneren Chordascheide eine mit Carmin sich intensiv färbende Masse gesehen und ist geneigt, diese Masse als ein natürliches Einschiebsel zwischen Chordascheide und Knorpel anzusehen, etwa als ein Ausscheidungsprodukt der Scheide. Dagegen will ich hervorheben, dass bei meinem gut gefärbten Embryo sich diese Masse $\mathrm{nicht}$ findet und dass wir diese Schicht morphologisch an dieser Stelle nicht verstehen können. Da schliesslich Froriep keine anderen Bedenken gegen die Auffassung dieser Masse als Gerinsel äussert als ihre Anziehungskraft für Farbstoff, so steht nichts im Wege, hier ein $\mathrm{Kunstprodukt}$ anzunehmen, von denen es ja bekannt ist, dass sie von vielen Farbstoffen auf das prächtigste gefärbt werden.

\section{Verlauf der Chorda.}

Die Chorda findet sich in der Wirbelsäule in der Mitte der Wirbelkörper und der Zwischenwirbelscheiben; Anschwellungen in diesen können wir nicht erkennen, die Dicke des Stranges bleibt ziemlich unverändert. Wie zu erwarten, sehen wir die Chorda im Zahn des Epistropheus, in den sie sich continuirlich aus dem Körper des Epistropheus fortsetzt. Der $\mathrm{Zahn}$ des Epistropheus verbäl sich zur Chorda genau wie e in Wirbelkö r per und erzählt so noch deutlich die Geschichte seiner Herkunft. Nachdem die Chorda den Zahnfortsatz ver. lassen, finden wir sie in einem kurzen.Strang dichten Bindege- 
webes, dem späteren Ligamentum suspensorium dentis, von wo aus wir die Regio occipitalis des Primordialcraniums erreichen. Hier ändert sich das Bild. Während die Chorda der Halswirbelsäule ziemlich senkrecht verläuft und auf jedem Querschnitte des Objectes ebenfalls quergeschnitten getroffen wird, passirt sie die Occipitalregion in dorsoventraler Richtung; wir finden sie also hier auf den Objectquerschnitten im Längsschnitt getroffen. Ihr Durchmesser ist hier sowohl seitlich als auch von oben nach unten verbreitert.

Nachdem die Chorda die ventrale Seite der Occipitalregion erreicht hat, verlässt sie dieselbe und damit zugleich vorläufig überhaupt das Primordialcranium. Sie befindet sich jetzt im retropharyngealen Raum und bildet hier eine starke Anschwellung. Beim näheren Zuschanen erkennen wir, dass die Anschwellung in einem stark geschlängelten Verlauf besteht, wie daraus hervorgeht, dass wir inmitten der Anschwellung mehrfach die Chordascheide vorfinden. Ventral erreicht die Anschwellung die Rachenwand, die hier eingestülpt ist und die Bursa pharyngea bildet. Auch ich habe, wie Froriep, nur eine sehr nahe Aneinanderlagerung der Chorda und des Rachenepithels gesehen, kann dagegen auch für mein Object eine Berïhrung nur für äusserst wahrscheinlich erklären. An dieser Stelle mögen auch noch starke Züge von Bindegewebe Erwähnung finden, welche zu beiden Seiten der Chorda vom Occipitalknorpel zur Rachenwand ziehen. (F rorieps Ligamenta pharyngo-occipitalia.)

Weiterhin strebt die Chorda wieder in verticaler Richtung scheitelwärts, erreicht das Primordialcranium wieder im Dorsum ephippii, in dem sie eine kurze Strecke vor dem freien Rande ihr Ende erreicht. Ausserdem finden wir aber vor der Sella turcica einen Rest der Chorda, indem wir sie hier von der Höhe des hinteren Endes eine kurze Strecke im Knorpel abwärts steigen sehen. Zwischen beiden Chordasträngen trifft man auf dem Knorpelboden der Sella turcica Bindegewebe, auf welchem erst in einem etwas höheren Niveau die Hypophyse gelagert ist.

\section{Regiones petrosae.}

Die Regiones petrosae dominiren im Primordialeranium durch ihre Grösse; sie thürmen sich im hinteren Bezirke des Craniums zu beiden Seiten der Spheno-occipitalregion auf. Mit 
Leichtigkeit lassen sich 3 Theile jeder Regio petrosa unterscheiden; ein kleiner, medial und vorn gelegener, die knorplige Schnecke (Fig. 1, Cchl.), lateral von ihr eine viel grössere Kapsel, welche die knorpligen Bogengänge birgt. Hinter und über diesen Kapseln finden wir schalenförmig gebogene Platten, die, wie wir später zeigen werden, von den Autoren bei den Säugern in bei weitem besserer Ausbildung beschrieben werden.

Die Schneckenkapsel hat etwa die Gestalt eines Ellipsoids mit vertikaler Hauptaxe; sie ist bis auf einige an der lateralen Seite gelegenen Oeffnungen völlig geschlossen. Durch die obere, die Hauptöffnung communicirt die häutige Schnecke mit den anderen Theilen des Labyrinthes und hier treten auch die Nervenfasern in die Kapsel ein.

Der Schneckenknorpel hat vielfache Verbindungen mit den Nachbarknorpelanlagen. Etwas uber seinem unteren Pole entsendet er zum Hinterhauptsknorpel eine knorplige Brïcke, in höheren Partieen legt er sich ihm ganz an, ohne jedoch mit ihm zu verschmelzen; das mikroskopische Bild lässt deutlich die Grenzen erkennen. Ebenfalls ohne Verschmelzung schliesst sich der Schneckenknorpel an die laterale Kante des Clivus an. Von der Vorderfläche der Kapsel zieht eine kleine Knorpelbrücke hinüber zu der Seitenwand des Keilbeinkörpers. Wir können an ihr zwei Portionen trennen, die vom Keilbeinknorpel und vom Schneckenknorpel aus entspringen und durch das Alter und die Histologie des Knorpels zu unterscheiden sind. Wie fruher erwähnt wurde, entspringt von dieser Brücke die Ala temporalis (A.t.) und zwar von der Portio sphenoidalis.

Durch diese Brïcke, den Keilbeinknorpel und die Schneckenkapsel wird ein Dreieck, der Vorläufer des Foramen lacerum anterius ( $I$ und $I I$ ) abgegrenzt, durch das jederseits die Carotis interna das obere Niveau der Schädelbasis erreicht, um sich dann längs des Keilbeins nach vorne $\mathrm{zu}$ wenden und schliesslich zu beiden Seiten der Hypophyse und des Zwischenhirns nach oben zu steigen. Lateral und hinten endlich geht die Schneckenkapsel ohne histologische Grenze in die benachbarte Abtheilung der Regio petrosa über.

Die \%weite Abtheilung der Regio petrosa hat ungefähr auch die Gestalt eines Ellipsoids, jedoch ohne sehr verschiedene Axen. 
Vorn und medial wird diese Abtheilung von der Schnecke begrenzt, unten vom Occipitalknorpel gestittzt. Oben und hinteu schliessen sich die schon erwäbnten grossen Knorpelplatten an, vorn und lateral sind Hammer und Ambos (Fig. 1 u. 2, H. u. A.) benachbart, nur durch das Perichondrium getrennt. Die Steigbügelanlage wird später besonders besprochen werden.

Bevor wir jetzt an die genauere Schilderung des Labyrinthknorpels herantreten können, müssen wir erst die anderen hier in Betracht kommenden Organe besprechen, soweit wie sie für das Verständniss des Knorpels von Wichtigkeit sind.

Facialis, acusticus ect. Der VII. und VIII. Hirnnerv treten durch eine gemeinsame Oeffnung (Fig 1, P.a.i.) in lateralventraler Richtung in den Knorpel ein. Der N. facialis wendet sich dann, indem er in seinem Verlaufe die Grenze zwischen der Schnecke und der lateralen Labyrinthkapsel darstellt, direkt nach vorn, verlässt hier durch eine schmale Knorpelliucke bereits wieder die Kapsel (VIII), bildet unmittelbar nach seinem Austritt das Ganglion geniculi und wendet sich dann lateral am Rande des Knorpels entlang. Wir finden ihn auf diesem Wege in der Nähe der Stapesanlage, sehen die Chorda tympani zu den Gehörknöchelchen herüberziehen und sehen ihn dann in die Tiefe steigen.

Der N. acusticus, der lateral vom Facialis in den Knorpel gelangt, bildet gleich nach seinem Eintritt zwei neben einander gelagerte Ganglien, das mediale Ganglion cochleare und das laterale Ganglion vestibulare. Von diesen Ganglien ziehen dann die Nervi vestibulares und cochleares hin zu ihren Endorganen im häutigen Labyrinth.

Das $h a ̈ u t i g e ~ L a b y r i n t h$ zerfällt noch nicht in ansgeprägter Weise in eine Pars superior und inferior, da die Sonderung in Sacculus und Utriculus noch in den ersten Anfängen ist; wir erkennen nur die Andeutung einer Falte an der medialen Labyrinthwand. Vor dieser Falte mündet mit 2 Armen der Re cessus labyrinthii, der mit deutlichem Lumen in die Höhe steigt, durch eine Spalte den Knorpel verlässt, in der freien Schädelhöhle eine blasenförmige Erweiterung bildet und blind endet. In der Nähe dieses völlig geschlossenen Endes sehen wir den Sinus transversus, der in einer Knorpelrinne emporsteigt.

Dic halbzirkelförmigen Kanäle und die häutige Schnecke 
habe ich nicht genau studirt, weil sie für mein Thema nicht in Betracht kommen.

Dic häutigen Labyrinththeile sind von embryonalem Bindegewebe umschlossen. In diesem Gewebe ist noch keine Differenzirung zu bemerken, höchstens finden wir im Verlaufe der Nerven eine dichtere Gewebsanordnung. Nach aussen geht das Bindegewebe in den Knorpel ubber; beim Sacculus und Utriculus ist es an der lateralen Seite etwa doppelt so breit als auf der medialen.

\section{Genaue Formverhältnisse des Labyrinthknorpels.}

Nachdem wir uns so vorgearbeitet haben, wird es möglich sein, die genaueren Formverhältnisse des Labyrinthknorpels zu erläutern. An der dorsalen Wand bemerken wir unten an der Grenze der Occipitalregion und der Regio petrosa einen länglichen breiten Spalt (Fig. 1,VI); durch ihn zieht lateral die Vena jugularis, medial die Nerven der Vagusgruppe. Die Abtheilungen des Spaltes sind durch einen vom Felsenbein vorspringenden Wulst getrennt, welcher auf der rechten Seite unseres Modells deutlicher ausgeprägt ist als links.

Viel höher, etwa in der Hypophysenhöhe, treffen wir eine Oeffnung in der Hinterwand der Labyrinthkapsel, durch die Facialis und Acusticus eintreten: den Porus acusticus internus (P.a.i.).

Etwas über dem Porus acusticus verlässt, wie bereits erwähnt, der Recessus labyrinthii durch eine Spalte, den A qua e ductus vestibuli, das Labyrinth (R.l.).

Ebenfalls haben wir schon auf die Knorpellücke (VIII) hingewiesen, durch die der Facialis das Labyrinth verlässt.

Auf die Anlage des Zitzenfortsatzes und die Umwandlung des obersten Endes des Reichert'schen Knorpels in den Processus styloideus brauche ich nicht einzugehen, da ich hier nichts unbekanntes zu berichten habe.

Von der Gegend des späteren Proc. mastoideus aus zieht aber nach vorne ein breiter Streifen dichteren Bindegewebes, welcher bis zur Anlage des Frontale reicht. v. Noorden hält diesen Streifen für eine knorplige Anlage der Squama temporis und stützt sich dabei anf v. Kölliker, der von einer rudimentären knorpligen Anlage der Squama temporis spricht. Bei meinem Object erinnert das Gewebe nicht an Knorpelanlagen, son- 
dern es ist im Gegentheil dem Bindegewebe gleichzustellen, aus dem sich der Deckknochen, z. B. das Frontale bildet.

\section{Stapesanlage.}

Um die Beschreibung des Labyrinthknorpels nicht unvollständig zu lassen, fügen wir noch einiges über die Stapesanlage hinzu. Am leichtesten orientirt man sich hier wohl durch Abbildungen.

Fig. 3. Die innere Randschieht der Labyrinthwand (L.), die sich von der ïbrigen Wand unterseheidet, besteht aus dichtem Bindegewebe, ausserdem aber bemerken wir in der Wand eine kleine, sich deutlich von der Umgebung abhebende Platte, das obere Ende der Steigbulgelanlage (St.), die noch aus Vorknorpel besteht. Von dem allgemeinen Knorpel der Labyrinthwand wird die Platte durch eine schmale Schicht Bindegewebe geschieden. Auf einer Reihe weiterer Schnitte (Fig. 4-7) erhalten wir noch deutliche Bilder der Steigbügelanlage $(S t$.), die allmählich frei im Bindegewebe liegt und weiter entwickelten Knorpel zeigt. In Fig. 5 ist die Knorpelplatte des Steigbligels von einem grösseren Loche durebbohrt, durch welches wir eine kleine Arteric, die Art. stapedia (A.st.) verlaufen sehen.

Für die folgenden Schnitte verweise ich auf $\mathrm{Baumgar-}$ ten's Beschreibung oder auf meine Abbildungen, mit deren Hülfe, wie ich hoffe, die Verhältnisse verständlich werden. Znsammenfassend ist zu sagen, dass wir durch Combination der Schnitte als Anlage des Steigbügels einen Ring erhalten, dessen Achse $45^{0}$ zur Vertikalebene geneigt ist. Ferner ist der Bindegewebsstreifen (Fig. 6 und 7, Lg.) nicht zu verkennen, der vom unteren Theile des Ringes zum Reichert'schen Knorpel zieht. Soweit wir die Stapesanlage nach unten verfolgen können, wird anch die Labyrinthwand nur durch eine dünne bindegewebige Schicht (Fig. 4-6, x.) gebildet. Was aus diesem Befund zu schliessen ist, werde ich später discutiren.

In Bezug auf $\mathrm{Hammer}$ und $\mathrm{Ambos}$ verweise ich auf Ba umgarten's Schrift.

\section{Meokel'sohe Knorpel (Mko).}

An die Betrachtung der Gehörknöchelchen knüpfe ich das Studium der M e c k el'schen Knorpel. Sie verlaufen, wie Sehnitte 
und Modell lehren (Fig. $2 \mathrm{Mk}$.), nach unten und ventral und verbreitern sich am distalen Ende zu je einer knorpligen Scheibe. Mit den so verbreiterten Enden legen sich die beiden Knorpel dicht zusammen und werden nur durch eine dünne Schicht von Bindegewebe von einander getrennt.

\section{Reichert'sche Rnorpel.}

Die R e i c h e r t'schen Knorpel, die wir schon bei der Stapesanlage erwähnt haben, lagern sich mit ihren proximalen Enden der Regio petrosa an, ohne jedoch mit ihr zu verschmelzen, verlaufen von hier nach abwärts und sind auf dem Niveau des Atlas unterbrochen, um erst wieder in mittlerer Zungenhöhe zu erscheinen und dann bis zu den Kehlkopfknorpeln herabzusteigen.

\section{Deckknochen.}

Die Betrachtung des Primordialcraniums wird cine vollständigere, wenn man zugleich feststellt, was an Deckknochen sich in der Gegend des Craniums bereits angelegt hat.

M a nd ibula (Fig. $2 M d b$.). Wir finden die ventrale Verbindungsstelle der beiden Meckel'schen Knorpel mit Knochenlamellen umgeben, die sich nach oben hin dem Knorpel folgend fortsetzen. Sie bilden zur Seite der Knorpel zwei Lamellen, welche dorsal zusammenstossen; zwischen den Lamellen verläuft der N. alveolaris. Nach oben hin setzt sich dann der Knochenstab in einer Lamelle fort, die immer dünner wird, $1 \mathrm{Im}$ in der Gegend der Gehörknöchelchen wieder dicker zu werden. Mit Recht vermutet $B$ a umgarten hier wohl die Anlage des Proc. folianus $(P . f$.$) . Die Frontalia (F r$.$) haben wir bei Gelegen-$ heit der Schilderung der Alae orbitales des Keilbeinknorpels schon beschrieben.

Die Maxillen (Mx.) sind bereits angelegt und zeigen, wie auch die Mandibula, topographische Beriehungen zu den Zalnanlagen, ohne dass man jedoch natlirlich etwa schon von Alveolen reden könnte.

Der Vomer endlich findet sich als p a a rige, unbedeutende Knochenanlage am unteren Ende der knorpligen Nasenscheidewand. 


\section{Allgemeiner Theil.}

Es wird jetzt meine Aufgabe sein zu berichten, welche Schlüsse sich aus meinen allerdings nur spärlichen Beobachtungen ziehen lassen und zugleich etwas auf die Literatur einzugehen. Ich werde das in folgenden Abschnitten thun.

I. Art und Weise des Verlaufes der Entwicklung.

II. Einige Beziehungen des menschlichen Primordialcraniums zu dem der Säuger.

III. Die Beurtheilung der Stapesanlage mit einigen Bemerkungen über die Meckel'schen Knorpel.

I.

Wenn wir uns zunächst mit der Art und Weise des Verlaufes der Entwicklung des Craniums beschäftigen, so ist hervorzuheben, dass die Ansichten in dieser Frage durch einen von Kölliker aufgestellten Satz beherrseht werden. Kölliker hatte auf Grund von Untersuchungen uber die Entwicklung des Primordialcraniums des $\mathrm{K}$ aninchens den Satz aufgestellt, dass das Cranium wie a us einem Gusse entstehe, nachher nur noch wachse, sich aber nicht weiter entwickele. Diese Thatsache wurde von den Autoren als allge me in gültig angesehen und anf die verschiedensten Säuger und auch auf den Menschen übertragen. Man hätte nicht so verfahren können, wenn man berücksichtigt hätte, dass das Kaninchen in der Zeit der Entwicklung seines Primordialcraniums d. i. in der Mitte seines intrauterinen Lebens, im allgemeinen eine sehr rasche und gedrängte Entwicklung erkennen lässt. Wir können uns daher denn auch nicht wundern, dass die Entwicklung des Primordialcraniums sich bei ihm auf eine sehr kurze Spanne Zeit zusammen drängt.

Von einer Verallgemeinerung des Befundes beim Kaninchen kann denn auch nicht die Rede sein. So lehren 'P arker's Untersuchungen über die Entwicklung des Primordialcraniums des Schweines eine Schritt für Schritt vor sich gehende Entwicklung. Dasselbe kann ich für den Mensehen bestätigen. Denn nicht nur v. Noorden's Stadien zeigten unter sich sehr erhebliche Entwicklungsdifferenzen, sondern auch mein Stadium war in seiner Entwicklung ein gut Theil weiter vorgeschritten als das älteste der v. Noorden'schen und an verschiedenen Stellen 
fand ich noch vorknorplige Anlagen als Zeichen einer noch weiter gehenden Entwicklung. Weiterhin ist ein von anderer Seite zu gleicher Zeit, im II. anatomischen Institute zu Berlin angefertigtes Modell eines menschlichen Primordialcraniums ein gutes Beispiel für eine noch weiter vorgeschrittene Entwicklung, für die auch noch Spöndli und Hannover als Zeugen anzuführen sind.

Auch der an sich richtige Satz, dass das Primordialcranium ein continuirliches Ganzes darstellt, ist zu weit ausgebeutet worden. So stimmt denn nicht, was diejenigen, die sich auf die Zergliederung beschränkt haben, behaupten, dass das knorplige Primordialcranium zu einer Zeit, in der noch keine Knochenkerne aufgetreten sind, durchaus keine Abgrenzung unter sich erkennen lässt, sondern vielmehr aneinander stossende Theile unvermittelt in einander übergehen. Ich schliesse mich am ehesten der Ansicht H a n nover's an, dass der Primordialknorpel des Mensehen eine zusammenbängende Masse ist, an der man zwar verschiedene Partieen, nicht aber getrennte Knorpelstücke unterseheiden kann. Ich finde einen Beweis für diese Behauptung in dem histolog is chen Verlualten, das an viclen Punkten deutliche Grenzen erkennen lässt.

\section{II.}

Bis vor einem Jahrzehnt finden wir bei den Autoren die Lehre, dass die Ausbildung des Primordialcraniums allmählich in der Reihe der Säuger abnehme und beim Menschen seinen niedrigsten Punkt erreiche.

De cker hat auf Grund seiner Untersuchungen an Sängethieren diesen Lehrsatz dahin abgeändert, dass sich für die Sänger keine continuirlich abnehmende, sondern eine schwankende Curve ergiebt. Meine Beobachtungen zeigen, dass das menschliche Primordialcranium cine bessere Ausbildung erkennen lässt als wie die Autoren sie fast durchweg an ihren Stadien gefunden haben. Ich habe hier besonders die Platten im Auge, die ich in mächtiger Ausbildung iiber dem Labyrinth gefunden habe. Von diesen Platten, welche von Kölliker und seinen Schülern Spöndli und D e cker Parietalplatten benannt werden, bemerkt Decker im allgemeinen, dass sie der Mittellinie entgegenwachsen, ohne sie jedoch, s o w e it be $\mathrm{k}$ a $\mathrm{n} \mathbf{n}$, zu erreichen.

Diese Parietalplatten, die bei einigen Säugern längere Zeit riesige Entwickelung zeigen, müssen beim Menschen meistens nur 
sehr vorübergehend eine gute Ausbildung erkennen lassen. Denn v. Noorden fand sie nur in ihren ersten Anfängen, während die Beobachter älterer Stadien, wie Spöndli, bereits wieder von der rudimentären Anlage der Platten reden. Bei meinem Objekt waren die Platten nicht nur seitlich stark entwickelt, sondern zeigten auch in den oberen Partieen einen dorsalen Verschluss. Die einzige Beobachtung, die in der ganzen Literatur, soweit ich es ubbersehen kann, vielleicht mit meinem Befund übereinstimmt, ist von Bessel-Hagen in einer vorläufigen Mittheilung zur Kenntniss gebracht worden. Auch der Schlussfolgerung Hagens, dass wir in diesen gut ausgebildeten, aber vergänglichen Gebilden eine Vermittlung zwischen dem menschlichen Primordialschädel und dem ausgedehnten Chondrocranium anderer Säuger zu suchen haben, glaube ich mich anschliessen zu können.

$\mathrm{Ja}$ ich meine, es ist sogar im menschlichen Knorpelcranium die Erinnerung daran gewahrt geblieben, dass in der Phylogenie eine knorpelige Verbindung zwischen den Alae orbitales Fig. $1 \mathrm{u}$. 2 Ao. und den Parietalplatten bestanden hat. Ich habe nämlich an den Alae orbitales ausser den Processus clinoidei anteriores $(I V)$ noch je einen Fortsatz ( $V$ ) beschrieben, der von der Stelle der stärksten Krümmung aus nach hinten und oben strebt. Dieser Processus entspricht keinem definitiven Gebilde des menschlichen Schädels; dagegen finden wir ihn in dem Primordialcranium der verschiedenen Säuger verschieden stark ausgebildet, ja bei manchen erreicht er sogar nach hinten die Parietalplatte. Es ist daher wohl sein rudimentäres Auftreten beim Menschen im Sinne eines phylogenetischen Restes aufzufassen.

Weiterhin war es von Interesse, dass wir die Alae temporales $(A, t$ ) des Keilbeinknorpels in geringerer Ausbildung als die Alae orbitales fanden, eine Thatsache, die bereits SpöndIi erwähnt. Ge ge n b a u r bezeichnet das Verhalten beim e r w a c hsenen Menschen als eine Eigenthümlichkeit des Menschen. Er hebt hervor, dass bei vielen Sängern die Alae temporales nicht grösser, sondern sogar kleiner als die Alae orbitales wären. Es ist also gewiss bemerkenswerth, dass im Primordialcranium des Menschen Verhältnisse bewahrt sind, die in der Thierwelt während des ganzen Lcbens persistiren.

\section{III.}

Wir waren an einer früheren Stelle bei der Schilderung der Archiv f. mikrosk, Anal. Bd. 44 
Befunde, welche die Schnitte durch die Stapesanlage ergaben, nicht auf die Schlüsse eingegangen, welche sich aus ihnen etwa ziehen lassen. Das soll im folgenden im Zusammenhang geschehen.

Es ist heutzutage wohl noch nicht möglich, von einem allgemeineren, vergleichenden Standpunkte die Frage nach der Herkunft des Stapes zu beantworten. Dazu sind die Grundlagen, die das Studium der Amphibien liefern muss, noch nicht genügend befestigt. Andrerseits ist für die Amnioten selbst vielfach betont worden, dass der R e i c h e r t'sche Knorpel bei der Stapesbildung betheiligt ist, sogar schon sehr frühzeitig, da ja auch bereits für $\mathrm{H}$ a t teri a dieser Zusammenhang nachgewiesen worden ist. Bei den Sä u gern haben Parker und besonders Gruber sich für die Entwickelung aus der Labyrinthwand ausgesprochen, während eine Reihe anderer Forscher den zweiten Kiemenbogen heranziehen. Die Betheiligung des ersten Kiemenbogens ist nach meiner Meinung überhaupt nicht mehr discutabel.

In neuerer Zeit hat nun Gra d e n i g o die Ansicht vertreten, dass beim Menschen der Stapes sich a u d e m z w ei te n B o gen und der Labyrinthwand entwickelt.

Zunächst glaube ich, kann die Beziehung des Stapes zum Reichert'schen Knorpel als einigermaassen gesichert gelten, da die Beobachtungen, namentlich von Gradenigo in dieser Bezichung ibberzeugend sind und namentlich durch $\mathrm{R}$ a bl's wichtigen Fund bezüglich der Nervenversorgung gestiitzt sind. Ferner hat sich auch B a mgarten, dessen Material und Modelle mir vorlagen, dahin ansgesprochen und ich kann mich ihm hierin anschliessen ${ }^{1}$ ). Andererseits glaube ich, muss noch betont werden, dass keiner von den Autoren, welche einen anderweitigen Ursprung des Stapes beobachtet haben, den Beweis erbracht hat, dass der Hyoidbogen $\mathrm{nicht}$ betheiligt ist.

1) Inzwischen hat noch $Z$ on dek neue und wie mir scheint, beachtenswerthe Belege für die Beziehung des Steigbügels und des Reichert'schen Knorpels beigebracht. (Beiträge zur Entwicklungsgeschichte der Gehörknöchelchen. Inaugural-Dissertation. Berlin, 1893.) - Sehr bemerkenswerth ist auch der neuerdings von Dreyfuss (Beitrïge zur Entwicklungsgeschichte des Mittelohres u. d. Trommelfells des Menschen u. d. Säugethiere. Morph. Arbeit. II. Bd. 1893) eingenommene, vorsichtige Standpunkt, da dieser Autor auf Grund reichen Materiales dazu gelangt, keine der bisher vertretenen Abstammungsweisen für vorläufig erwiesen anzusehen. 
Während also die Betheiligung des $\mathrm{R}$ e i c h e r t'schen Knorpels vielleicht gesichert sein dürfte, so ist bei der Labyrinthwand die Sachlage verwickelter. Denn Gra d en i g o's Befunde lassen noch den Einwand zu, dass die Stapesplatte erst secundär in die Labyrinthwand eingelassen worden ist und auch der B a u m garte n'sche Embryo, den ich nachgeprüft habe, zeigt, wie ich glaube, dieses Verhältniss. Die Entscheidung über die Abstammung des Stapes von der Labyrinthwand muss nach dem Studinm jüngerer Stadien getroffen werden. Und hier steht v. Noorden's positiver Befund, der sicherlich von grossem Interesse ist, bisher wenigstens zu vereinzelt da und sind die betreffenden Angaben nicht bestimmt genug, um überzeugen zu können. Es bleibt also die Frage noch offen, da sowohl die vergleichende Anatomie als anch die Entwicklungsgeschichte noch nicht das letzte Wort gesprochen haben.

Fügen wir noch einiges hier über die $d$ istalen Enden der Meckel'schen Knorpel an, über die in der Literatur Unsicherheit herrscht. Im allgemeinen wird überwiegend die Ansicht vertreten, dass bei verschiedenen Säugern eine Verbindung der distalen Enden statt hat, beim $\mathrm{Mens}$ chen aber nicht. Während $\mathrm{Meckel}$ selbst von einem menschlichen Embryo berichtet hatte, dass sich der Knorpelstreifen mit dem der anderen Seite bisweilen, vielleicht immer unter einem spitzen Winkel vereinige, ist Dursy's abweichende Beobachtung später allgemein angenommen worden und auch in die Lehrbücher übergegangen. Nach Dursy sind die distalen Enden der Meckel'schen Knorpel beim Menschen durch eine breite Fasersehicht von einander geschieden. Ich fand nur eine ganz schmale Perichondriumschicht zwischen den Knorpeln, wie es ähnlich anch Gradenigo gesehen hat. Dursy's abweichende Angaben erklären sich dadurch, dass er nur Frontalschnitte, die für diese Frage ungünstig sind, studirt hat und er noch nicht den Vortheil der Serienbetrachtung sich zu Nutzen machen konnte.

Ich habe auch die Untersuchung der ersten Anlage des menschlichen Primordialcraniums in Angriff genommen, werde darïber aber erst später berichten, da mein Material noch nicht für diese Frage vollständig genug ist. 


\section{Literatur.}

1. Baumgarten, Beitrïge zur Entwicklungsgeschichte der Gehörknöchelchen. Arch. f. mikrosk. Anatomie. Bd. 40.

2. Bessel-Hagen, Vorläufige Mittheilung über die Entwicklungsgeschichte des menschlichen Occiput und der abnormen Bildungen. des Os. occipitis. Monatsbericht d. kgl. preuss. Akad. d. Wissensch. zu Berlin, 1879, p. 267.

3. Decker, Ueber den Primordialschädel einiger Süugethiere. Zeitschrift f. wissensch. Zoologie. Bd. 38, 1883.

4. Dugès, Recherches sur l'osteologie et la myologie d. Batraciens à leurs differents âges. 1834.

5. Dursy, Zur Entwicklung'sgeschichte des Kopfes des Menschen und der höheren Wirbelthiere. Tübingen, 1869.

6. Froriep. Zur Entwicklungsseschichte der Chorda dorsalis bei menschlichen Embryonen. Beiträge aus d. Gebiete d. Anatomie u. Embryologie; als Festgabe Jakob Henle dargebracht von seinen Schülern. Bonn, 1882.

7. Derselbe, Zur Entwicklungsgeschichte der Wirbelsäule, insbes. des Atlas und Epistropheus und der Occipitalregion. II. Beobachtung an Säugethierembryonen. Arch. f. Anatomie u. Physiologie. Anatomische Abtheilung, 1886.

8. Gaupp, Primordialcranium und Hyoidbogen von Rana fusca. Habilitationsschrift d. Breslauer medic. Facult. 1893. Abdruck aus Morphol. Arb. 1893. Herausg. v. Schwalbe.

9. Gegenbaur, Das Kopfskelet der Selachier. Ein Btrg. z. Erkenntniss d. Genese d. Kopfskelets d. Wirbelthiere. Leipzig, 1872.

10. Derselbe, Ueber primäre und sekundäre Knochenbildung. Jenaische Zeitschrift. Bd. III.

11. Derselbe, Lehrbuch der Anatomie des Menschen. 4. Aufl. 1890.

12. Gradenigo, Die embryonale Anlage des Mittelohres: Die morphologische Bedeutung der Gehörknöchelchen. Mitthḷ. a. d. embryolog. Inst. d. Univers. Wien, 1887.

13. Gruber, Beitrag zur Entwicklungsgeschichte des Steigbügels und ovalen Fensters. Monatsschr. f. Ohrenheilk. No. 12 (s. auch Mitthl. aus d. embryolog. Inst. d. Univers. Wien, 1877 u. Bericht d. 50. Naturforscherversammlung. München, 1877).

14. Derselbe, Zur Entwicklungsgeschichte des Hörorgans der Süugethiere und des Menschen. Monatsschr. f'. Ohrenheilk. 1878.

15. Hannover, Primordialbrusken og dens Forbening i det meneskelige Cranium for Fodselen. (Table des matières et explication des planches en français.) Vidensk. Selsk. Skr. Bäkkes 5 naturviddenskabelig og mathematisk. Af. V 6. Kjobenhavn 1880. 
16. Derselbe, Primordialbrusken og dens Forbening in Truncus og Extremiteter hos Mennesket for Fodselen. Vidensk. Selsk. Skr. Bäkke 6 nat. of. d. IV. 3.

17. Derselbe, Le cartilage primordial et son ossification dans le crâne humain avant la naissance. Copenhague, 1881.

18. Hertwig, O., Ueber das Zahnsystem der Amphibien und seine Bedeutung für die Genese des Skelets der Mundhöhle. Arch. f. mikrosk. Anatomie. Bd. XI, Suppl. 1874.

19. Derselbe, Lehrbuch der Entwicklungsgeschichte des Menschen und der Wirbelthiere. IV. Aufl. 1893.

20. His, Anatomie menschlicher Embryonen. Leipzig, 1880-85.

21. Huxley, On the Representatives of the Malleus and the Incus of the Mammalia in the other Vertebrata. Proceedings of the Royal Society, 1869.

22. v. Kölliker, Allgemeine Betrachtungen über die Entstehung des knöchernen Schädels der Wirbelthiere. Bericht v. d. kgl. zootom. Anst. zu Würzburg, 1849.

23. Derselbe, Lehrbuch der Entwicklungsgeschichte. 1861 und 1879.

24. Meckel, Handbuch der menschlichen Anatomie. 4. Bd.: Besondere Anatomie, Eingeweidelehre und Geschichte des Fötus. Halle und Berlin, 1820.

25. v. Noorden, Beitrag zur Anatomie der knorpligen Schädelbasis menschlicher Embryonen. Arch. f. Anatomie u. Physiol. Anatom. Abtheil. 1887.

26. Parker, W. R., On the Structure and Development of the Skull in the Pig. Philos. Transact. 1874.

27. Derselbe und Bettany, Die Morphologie des Schädels. Uebers. von Vetter. Deutsche Ausgabe, 1879.

28. Rabl, Ueber das Gebiet des Nervus facialis. Anatom. Anz. 1887.

29. Rathke, Entwicklungsgeschichte der Natter. Königsberg, 1839.

30. Derselbe, IV. Bericht über das naturwissenschaftliche Seminar zu Königsberg nebst einer Abhandlung über die Entwicklung des Schädels der Wirbelthiere. Königsberg, 1839.

31. Reichert, Ueber die Visceralbogen der Wirbelthiere im Allgemeinen und deren Metamorphose bei den Säugethieren und Vögeln. Berlin, 1837.

32. Derselbe, Der Bau des menschlichen Gehirns. 1859 u. 1861.

33. Spöndli, Ueber den Primordialschädel der Säugethiere und des Menschen. Inaug.-Dissert. Zürich, 1846.

34. Stöhr, Zur Entwicklungsgeschichte des Urodelenschädels. Zeitschrift f. wissensch. Zoologie. Bd. 33.

35. Derselbe, Zur Entwicklungsgesch. d. Anurenschädels etc. Bd. 36.

36. Virchow, Zur Entwicklungsgeschichte des menschlichen Schädelgrundes. Berlin, 1857.

37. Wi ed ersheim, Kopfskelet d. Urodelen. Morph. Jahrb. Bd. III, 1877.

38. Derselbe, Grundriss der vergleichenden Anatomie. Jena, 1888. 
86 M. Ja coby : Ein Beitrag z. Kenntniss d. menschl. Primordialcraniums.

\section{Erklärung der Abbildungen auf Tafel V.}

\begin{tabular}{|c|c|c|c|}
\hline \multicolumn{4}{|c|}{ Abkurzungen. } \\
\hline$I$ & $\begin{aligned}= & \text { Foramen laceum an- } \\
& \text { terius. }\end{aligned}$ & $\begin{array}{l}\text { Cl. B. } \\
\text { Cr.g. }\end{array}$ & $\begin{array}{l}=\text { Clivus Blumenbachii. } \\
=\text { Crista galli. }\end{array}$ \\
\hline$H$ & $=$ Carotis interna. & D.e. & $=$ Dorsum ephippii. \\
\hline$I I I$ & $=$ Foramen opticum. & $F r$ & $=$ Frontale. \\
\hline$I V$ & $\begin{aligned}= & \text { Processus clinoideus } \\
& \text { anterior. }\end{aligned}$ & $\begin{array}{l}H \cdot u \cdot A \\
L .\end{array}$ & $\begin{array}{l}=\text { Hammer und Ambos. } \\
=\text { Labyrinthwand. }\end{array}$ \\
\hline$V$ & $=\begin{array}{l}2 . \text { Fortsatz der Ala } \\
\text { orbitalis. }\end{array}$ & $L g$. & $\begin{aligned}= & \text { Ligament vom Reichert' } \\
& \text { schenKnorpelzum Stapes. }\end{aligned}$ \\
\hline$V I$ & $\begin{array}{l}=\text { Canal für die Vagus- } \\
\text { gruppe und die Vena } \\
\text { jugularis. }\end{array}$ & $\begin{array}{l}M d b . \\
M . K . \\
M x .\end{array}$ & $\begin{array}{l}=\text { Mandibula. } \\
=\text { Meckel'scher Knorpel. } \\
=\text { Maxillare. }\end{array}$ \\
\hline$V I I$ & $\begin{array}{l}=\text { Rinne für den Sinus } \\
\text { venosus. }\end{array}$ & $\begin{array}{l}\text { Op. } \\
P . a . i\end{array}$ & $\begin{array}{l}=\text { Orbitalplatte(nachDursy) } \\
=\text { Porus acusticus internus. }\end{array}$ \\
\hline$V I I I$ & $\begin{aligned}= & \text { Austrittsstelle des } \mathrm{N} . \\
& \text { facialis. }\end{aligned}$ & $\begin{array}{l}\text { P.s. } \\
\text { R. } l .\end{array}$ & $\begin{array}{l}=\text { Processus folianus. } \\
=\text { Austrittsstelle für den Re }\end{array}$ \\
\hline$I X$ & $\begin{aligned}= & \text { Canalis pro nervo hy- } \\
& \text { poglosso. }\end{aligned}$ & $R . n$. & $\begin{aligned} & \text { cessus labyrinthii. } \\
= & \text { Regio nasalis. }\end{aligned}$ \\
\hline A. 0 . & $=$ Alae orbitalis. & R.p. & $=$ Regio petrosa: \\
\hline & $=$ Arteria stapedialis. & St. & $=$ Stapesanlage. \\
\hline A.t. & $=$ Ala temporales. & S.t. & $=$ Sella turcica. \\
\hline$C \operatorname{chl}$ & $=$ Cochlea. & T.e. & $=$ Tuberculum ephippii. \\
\hline
\end{tabular}

Ch. n. opt. = Ort des Chiasma nerv.

opt.

Menschlicher Embryo (30 mm S. S.-lïnge).

Fig. 1. Reconstructionsmodell des Primordialeraniums. 25fache Vergrösserung. Die Zeichnung wurde in $2 / 3$ Grösse angefertigt und nachher vom Lithographen um $1 / 5$ verkleinert. Von oben gesehen. Die Deckknochen gelb.

Fig. 2. Die eine Hälfte desselben Modells, von der Seite gesehen. Fig. 3-8. Schnitte durch die Stapesanlage. Schematisirt Zeiss A. 1. Abbe'scher Zeichenapparat. Knorpelanlage mit Tusche angelegt. Bindegewebsanlage punktirt. 

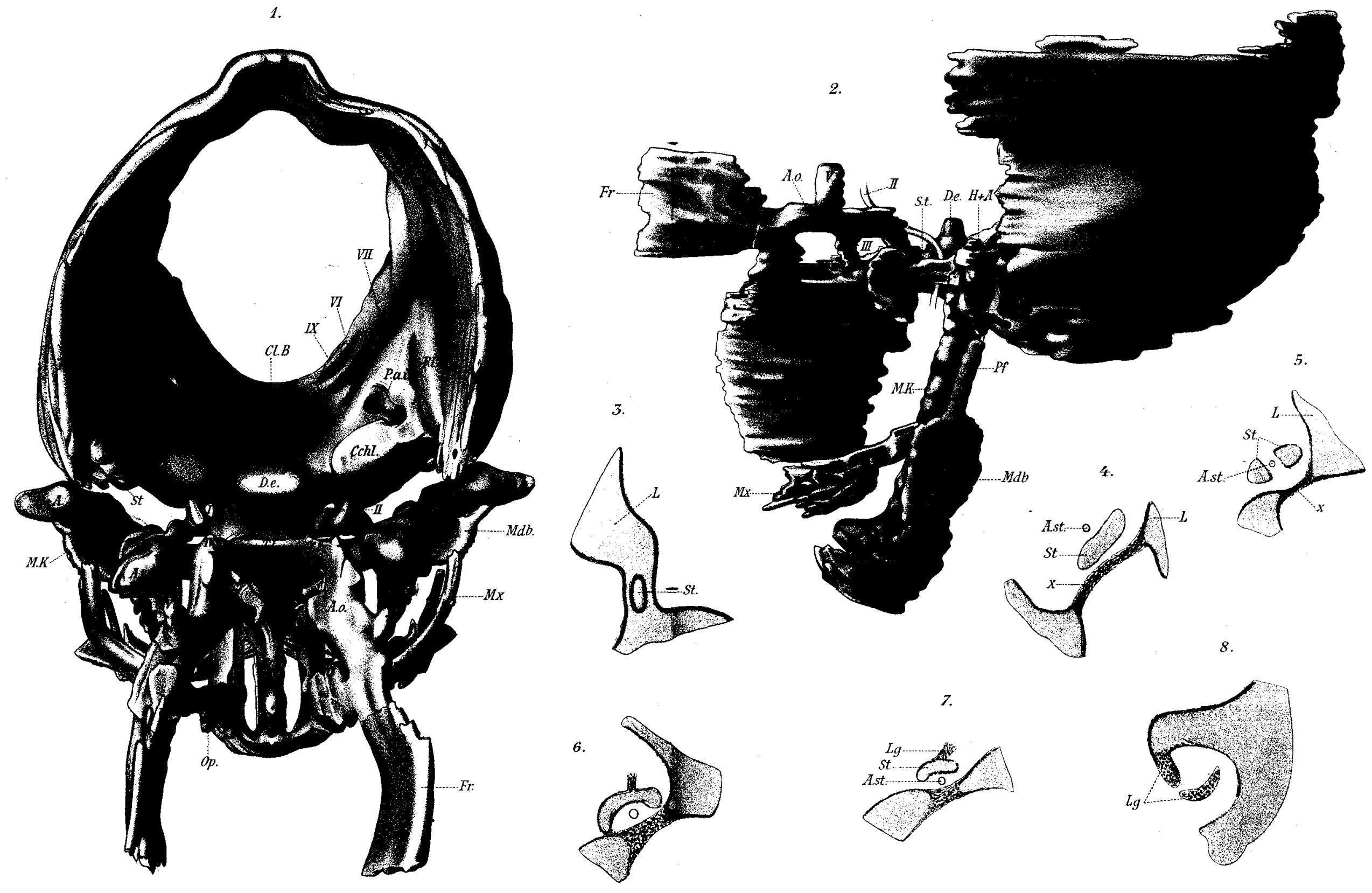